Dawson, B., Farnworth, E. H., McLeod, J. W. \& Nicholson, D. E. (1951). J. gen. Microbiol. 5, 408-415.

\title{
Observations on the Value of the Bordet-Gengou Medium for the Cultivation of Haemophilus pertussis
}

\author{
BY BETTY DAWSON, ENID H. FARNWORTH, J. W. MCLEOD \\ AND D. E. NICHOLSON
}

School of Medicine, Leeds

\begin{abstract}
SUMMARY : No significant improvement has been made on the medium first described for the primary culture of Haemophilus pertussis by Bordet \& Gengou in 1906. An explanation for this was sought in the experiments described, and it was found to be most probable that potato extract provides optimal concentrations of amino-acids and peptides required for growth. Although serum can be used to replace whole blood in this medium, the latter is essential for maximal growth; for that reason the retention of the term haemophilus is justified.

Many peptones, especially the more digested ones, inhibit growth of $H$. pertussis in concentrations normally used in bacteriological media. In the absence of peptone, meat extract agar can be used as the base of a satisfactory medium, especially when used in conjunction with horse instead of rabbit blood.
\end{abstract}

In surveying the literature on the cultivation of $\boldsymbol{H}$. pertussis we have not found any record of a considerable series of isolations of the bacillus from cases of the disease in which authors have departed seriously from the original medium of Bordet \& Gengou (1906) (cf. Wollstein, 1909; Chievitz \& Mayer, 1918; Sugare \& McLeod, 1929; Sauer \& Hambrecht, 1930; Gardner \& Leslie, 1932; Straker \& Westwater, 1937). A minor divergence from the original formula which has sometimes been used is the incorporation of c. $0.5 \%$ of lactic acid in the potato-extract blood agar, e.g. Leslie \& Gardner (1931) following a recommendation of the Danish school (Madsen, 1925). Bailey (1933) recommended a similar modification and maintained that such a medium gave better growth than the original Bordet-Gengou medium (referred to subsequently in this paper as BG medium).

We have seen only one other claim to have prepared a medium for phase I strains which is better than the original, that of Silverthorne \& Cameron (1942), who recommended a beef-liver extract blood agar. In our experience, however, this is no better than the standard BG medium. Surprisingly some text-books, e.g. Mackie \& McCartney (1949), recommend a medium which differs from the original BG medium in the important respect that it contains peptone. Its success probably depends on the facts that the peptone recommended is one of the least inhibitory (see Table 1), and that in the complete medium it is only present in $0.5 \%(\mathrm{w} / \mathrm{v})$ concentration. Pollock (1947) reported that 'the modified (BG) medium most commonly used consists of $30-50 \%$ whole blood and potato extract as well as a basis of peptone or tryptic digest of meat'.

It has frequently been observed, although insufficiently emphasized in some text-books, that freshly-isolated phase I strains of $\boldsymbol{H}$. pertussis fail to grow or grow very poorly on nutrient agar even with the incorporation of blood. This is stated in the work of Kristensen (1922), of Sugare \& McLeod (1929), of 
Kleinschmidt (1931) who observed that recently isolated $\boldsymbol{H}$. pertussis did not grow on Levinthal agar, and of Lawson (1939) who pointed out that smooth phase I $\boldsymbol{H}$. pertussis did not grow on chocolate agar, Fildes agar, blood agar, ascitic agar, or Loeffler's medium.

In our experience, an organism of correct morphology and staining which grows well on BG medium and fails to grow on heated blood nutrient agar ('chocolate') is quite as accurately recognized as a smooth phase I strain of $H$. pertussis as by agglutination. This is in frank contrast with $H$. influenzae, $\boldsymbol{H}$. parapertussis and $\boldsymbol{H}$. pertussis in other phases. So far as we have been able to determine, no one has explained this phenomenon. Pollock (1947) made interesting observations on factors controlling the growth of $\boldsymbol{H}$. pertussis and demonstrated its special sensitivity to unsaturated fatty acids and the significance of serum albumin in protecting the bacterium from the noxious effect of these fatty acids. His work, however, was done with a partly adapted or trained strain of $\boldsymbol{H}$. pertussis which was capable of growing on $10 \%$ blood agar. Further, in our hands the incorporation of activated charcoal in nutrient agar did not promote the growth of very recently isolated strains of $H$. pertussis, as Pollock found that it did that with his adapted strain. Hence his observations do not explain the failure of recently-isolated strains of $\boldsymbol{H}$. pertussis to grow on blood nutrient agar. They may explain the peculiar discrepancy that the fluid medium of Hornibrook $(1939,1940)$, which has been extensively used to obtain growths of $\boldsymbol{H}$. pertussis, fails when used as a solid agar medium (Verwey, Thiele, Sage \& Schuchardt, 1949). According to Pollock, agar itself may contribute unsaturated fatty acids.

The principal objective in our work was to find a medium which would promote more abundant and rapid growth of $H$. pertussis than does BG medium, and which would, therefore, afford a more rapid and constant aid in the diagnosis of whooping-cough. We decided, therefore, to survey each of the ingredients in the BG medium, and to search for an explanation of the peculiar difficulty of growing recently-isolated strains in nutrient agar with added blood. The results of our work may be summarized by stating that for the purpose of growth from small inocula of strains recently isolated ( $\frac{1}{2}-3$ weeks), we failed to find any medium which consistently gives better growths than the original BG medium prepared with rabbit or human blood. Our work, however, revealed certain facts with regard to the primary culture of $\boldsymbol{H}$. pertussis which have been insufficiently emphasized.

The original BG medium consisted of a base of potato extract, glycerol and saline, to which $50 \%(\mathrm{v} / \mathrm{v})$ whole blood was added. In the original communication rabbit or preferably human blood was recommended. This formula is the basis for most preparations of BG medium to-day, although the proportion of blood is usually only $30 \%$ and bloods from other species (e.g. sheep, horse) are used. It should be observed that the original BG formula does not require any peptone. This is definitely stated (Bordet \& Gengou, 1906; footnote to p. 734), but no reason is given other than that there is less profuse growth of saprophytic bacteria in its absence. The relevance of this omission of peptone will be stressed later. 


\section{EXPERIMENTAL}

At least three strains were tested simultaneously in all experiments, and invariably one or more of these strains had been isolated within a fortnight of the experiment and had undergone at the most two subcultures on BG medium. In this respect there is a sharp contrast with much other work on the subject. Approximately standard inocula were obtained by preparing 1/100 and 1/1000 dilutions of suspensions of just visible turbidity in saline. One loopful of each of the dilutions was used, and it was found that from the more dilute inocula a countable number of discrete colonies grew on suitable media, so that the size and numbers of colonies could be compared. One or more control plates of BG medium were included in every experiment. Each component of the BG medium was then examined to determine its importance.

Glycerol. The value of the glycerol does not appear to be very great. Although normally added to the potato before extraction, addition at a later stage does not appear to be a disadvantage. Increase in concentration beyond $1 \%(\mathrm{w} / \mathrm{v})$ in the basal medium produces some inhibition of growth, while its complete elimination causes only slight diminution of growth. In all control plates of BG medium, however, it was retained.

Agar. Variations in media caused by the use of different commercial preparations of agar suggested that some were definitely superior to others. A sample of ' Oxoid' agar (code no. L. 10) prepared by Oxo Ltd. London, from material of New Zealand origin, was satisfactory and used throughout at $1.4 \%(w / v)$ in the completed medium.

Potato extract. In this complex component only the most obvious constituents have been examined. An average analysis of whole potato gives carbohydrate $20 \%$ and protein $2 \%$. Soluble carbohydrate, protein or protein derivatives and inorganic salts are present in the aqueous extract. The effects of extracts of different parts of the potato, of different varieties of potato, and of different methods of preparation were studied. The following extracts were incorporated in BG medium and gave similar growths: from cortex or centre of young, old and sprouting potatoes or of varieties obtained from different areas. Equally good growth was obtained when the extract was prepared by autoclaving slices after standing in water for $\mathbf{3 0}$ min., or by dropping them into boiling water as soon as sliced. Filtration of an extract of autoclaved potato gave as good a result as unfiltered extract containing much insoluble matter. It appears that considerable variations in the preparation of potato extract may be adopted without appreciable effect. There are variations, however, which result in definitely inferior growth. This occurs when the extract is concentrated to half its usual volume, when potato starch solution is substituted for it, or when extracts at temperatures below $55^{\circ}$ are sterilized by filtration with Maassen candles. The last procedure, designed to retain active enzymes, yielded extracts which partially inhibited growth and became dark brown on standing. The method of extraction finally adopted was to autoclave thinly-sliced peeled potato at $10 \mathrm{lb}$./sq.in. for $15 \mathrm{~min}$., with twice its weight of aqueous glycerol (4\%). The extract was then separated by filtration through lint, using 
moderate pressure, and diluted with $0.6 \%$ saline in accordance with the BG formula.

\section{Observations on the amino-acid content of potato extract}

The filtrates and residues obtained both by dialysis and by ultra-filtration using collodion sacs were compared with the original extract. This comparison showed that the growth-promoting material passed through the bag, the dialysate and ultra-filtrate giving results identical with those obtained with the original potato extract. The residuum left after dialysis or ultrafiltration gave poorer growth. The protein and starch present in potato extract appear, therefore, to be relatively unimportant. Although Groot (1946) made an extensive investigation of the amino-acids present in potato protein this examination did not extend to the free amino-acids present in potato juice or extract. As we have not found any account of the amino-acid content of potato extract we examined it by two-way paper chromatography. There was evidence of the presence of many amino-acids, including glutamic and aspartic acids, which was interesting in view of the fact that these two amino-acids have a stimulating effect on the growth of $\boldsymbol{H}$. pertussis (see below). Since an acid-hydrolysate of casein equivalent to about $1 \%(\mathrm{w} / \mathrm{v})$ in the basal medium gave growth only slightly inferior to that obtained with potato extract it may be that a collection of amino-acids in certain proportions is responsible for the value of potato extract in the BG medium. It should be mentioned that $H$. pertussis will grow on a medium entirely devoid of potato extract, i.e. containing saline, agar and blood only, although growth is much less good.

\section{Blood}

Bordet \& Gengou originally suggested the incorporation of $50 \%(\mathrm{v} / \mathrm{v})$ human or rabbit blood in their medium. We confirmed that in their medium the bloods of these two species are superior to that of the horse. The amount required can be varied between 30 and $50 \%(\mathrm{v} / \mathrm{v})$ without appreciable change in the amount of growth; with less than $30 \%$ progressively inferior results were obtained. Defibrinated and oxalated blood were equally effective. It is stressed in some text-books that $\boldsymbol{H}$. pertussis may be differentiated from $\boldsymbol{H}$. influenzae by its inability to grow in primary culture on a heated blood medium. In these cases, however, it is probable that blood nutrient agar was in question. In our experience the heating of BG medium to $75^{\circ}$ to convert it to 'chocolate' gives growth only slightly inferior to that obtained on the unheated medium. By contrast the growth of $\boldsymbol{H}$. influenzae is greatly enhanced on the heated medium. Since Pollock (1947) emphasized the value of serum albumin, and Verwey et al. (1949) found that washed blood cells promoted growth in a medium which did not support growth without this addition, we examined the different constituents of blood.

Haemolysed blood. Rabbit blood haemolysed by alternate freezing and thawing was equivalent to unhaemolysed blood.

Serum. Good growth was obtained on a medium consisting of potato glycerol agar base to which serum was added. Horse, sheep, human or rabbit sera were 
satisfactory. The variation in species produced less variation of growth of $H$. pertussis than was obtained with whole blood, although rabbit serum was superior to horse serum. When different concentrations of serum were used the best results were obtained with 15-25 \% (v/v); $20 \%$ of serum was, therefore, chosen. In general, rabbit serum was used, and samples from a number of different varieties of rabbit appeared to be identical in effect. An adverse effect on the serum was obtained by heating at $75^{\circ}$, but not at $60^{\circ}$, and was much more pronounced than the effect of heating the complete BG medium at $75^{\circ}$.

Blood corpuscles. Although Verwey et al. (1949) reported that the addition of human blood cells to their partly defined medium produced a medium better than BG medium for growth from heavy inocula, we found that washed cells had very little value in promoting growth when used in making BG medium. The same batch of BG base which gave profuse growth with whole blood gave no discernible growth in 3 days when carefully washed red cells were used in equivalent amount. The washed red cells were ineffective whether haemolysed or not. Growth in serum agar was augmented by adding a solution of haemolysed washed red blood corpuscles, the increase being proportional to the amount added.

Effect of different samples of human blood. Four samples of blood from students who had had attacks of whooping-cough and four others from students with no history of whooping-cough were compared in BG medium; growth was good and identical with all samples.

It appears therefore, that the blood serum is a specially important component in BG medium; both large- and small-molecular constituents appear to be necessary since ultrafiltration of serum yields two relatively inactive parts (filtrate and residue) which, if recombined, promote growth almost as much as the unfractionated serum.

Peptone and amino-acids. It has already been stressed that peptone was not included in the original BG medium. Nevertheless, since it is often added, the effect of different brands of peptone in BG base was examined. Each of nine different commercial peptones was incorporated (at $1 \% \mathrm{w} / \mathrm{v}$ ) in potato extract glycerol agar base and after the addition of $30 \%(v / v)$ rabbit blood, plates were poured. On inoculation with two recently-isolated strains of $H$. pertussis in heavier and lighter concentrations the results recorded in Table 1 were obtained. Similar results were obtained whether the basal medium was adjusted to $\mathrm{pH} 7 \cdot 6$ or left without adjustment of reaction as in the original formula of Bordet \& Gengou. Table 1 shows a better result without peptone than with any of those added, and that Witte and Difco proteose peptones were less inhibitory than the others. Examination of these peptones showed that the Witte and Difco proteose peptones were much less hydrolysed than the others.

O'Meara \& Macsween (1937) and Balch (1942) drew attention to the varying copper content of peptones and the inhibitory effect of traces of this element on many delicate bacteria. We examined the sensitivity of $H$. pertussis to copper (as copper sulphate) in the BG medium. No inhibition was observed even with amounts of copper up to $2 \mu \mathrm{g} . / \mathrm{ml}$. It would appear, therefore, 
that $\boldsymbol{H}$. pertussis behaved on our medium like those Gram-negative bacteria which were observed by O'Meara \& Macsween.

In view of the possibility that the amino-acid content of the potato dialysate was important, other material containing amino-acids was examined. Since the average total $\mathrm{N}$ and amino- $\mathrm{N}$ content of peptone is about 12 and $2 \%$ respectively of the dry weight, a series of plates was made up in which casein hydrolysate was incorporated with BG medium in various concentrations about this range of nitrogen content. The product known as Casamino acids (Difco) was at first used. In concentrations of $0.1 \%$ some enhancement of

Table 1. Inhibition of growth of Haemophilus pertussis by various peptones

BG basal medium throughout. Different peptones $(1 \% \mathrm{w} / \mathrm{v})$ were added. Inocula: loopfuls from 1/100 and 1/1000 dilutions of faintly turbid suspension of $H$. pertussis.

\begin{tabular}{|c|c|c|c|c|}
\hline \multirow[b]{2}{*}{ Addition } & \multicolumn{2}{|c|}{ Strain I } & \multicolumn{2}{|c|}{ Strain II } \\
\hline & Heavy & Light & Heavy & Light \\
\hline $\begin{array}{l}\text { None (control) } \\
\text { Witte peptone }\end{array}$ & $\begin{array}{c}+++ \\
++\end{array}$ & $\begin{array}{c}++ \\
+\end{array}$ & $\begin{array}{c}+++ \\
++\end{array}$ & $\begin{array}{l}++ \\
+\end{array}$ \\
\hline Difco proteose peptone & + & + & + & + \\
\hline Oxo peptone & 3 colonies & - & - & - \\
\hline Parke Davis peptone & 1 colony & - & - & - \\
\hline Evans peptone & 1 colony & - & - & - \\
\hline Bacto-Difco peptone & 1 colony & - & - & - \\
\hline Difco tryptose peptone & 1 colony & - & - & - \\
\hline Vaillant peptone & 3 colonjes & - & - & - \\
\hline Eupeptone & 2 colonies & - & - & - \\
\hline
\end{tabular}

,,$+++++=$ numerous discrete colonies, partly confluent, confluent growth.

growth was produced, but at $1 \%$ there was partial inhibition and at or about $2 \%$ complete inhibition. A series of plates was prepared in which separate amino-acids were added in the proportions in which they were calculated to occur in $0 \cdot 1,1$ and $2 \%$ of casein hydrolysate. The results of this series of experiments were not always consistent, but certain effects emerged. Aspartic and glutamic acids tended to promote and serine tended to inhibit growth. Both effects could usually be demonstrated in concentrations of amino-acid of c. $0.05 \%$. The stimulant effect of aspartic acid was found to vary with the preparation used; a recent sample from our usual source of supply used in experiments not here recorded was darker than the original and was much inferior. The presence or absence of impurities in the available preparations of this amino-acid must, therefore, be taken into account by anyone who uses this substance.

\section{Substitutes for potato base in BG medium}

Clearly the inhibitory activity of some peptones might explain the failure of blood nutrient agar to support growth. It was, therefore, of interest to observe the effect of excluding peptone from that medium. Meat extract was prepared by simmering minced beef with twice its weight of water for $2 \mathrm{hr}$. in a glass vessel, and then filtering through lint and filter-paper. The extract

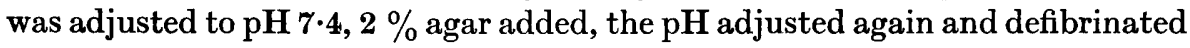


rabbit blood (30 \% v/v) added. This medium, although often inferior to BG medium, occasionally yielded better growths. When oxalated horse blood was used in place of rabbit blood a considerable improvement resulted and growth was obtained very similar in amount to that on BG medium and superior to BG medium prepared with horse blood.

In this meat extract horse blood medium the effect of additions of aminoacids and peptones was similar to and often more marked than that in BG medium. With aspartic acid an effect of some interest was observed. Plates of BG medium $+0.05 \%$ aspartic acid were inoculated not only with strains of $\boldsymbol{H}$. pertussis but also with $\boldsymbol{H}$. influenzae, and although in general the action of amino-acids on the growth of $\boldsymbol{H}$. influenzae was not inhibitory, aspartic acid checked its growth distinctly. Incorporation of this amino-acid in a medium for isolation of $\boldsymbol{H}$. pertussis would presumably have the two-fold advantage of somewhat promoting growth of $\boldsymbol{H}$. pertussis and inhibiting that of $\boldsymbol{H}$. influenzae. In addition, horse blood instead of rabbit blood exerted a further inhibitory action on some strains of $\boldsymbol{H}$. influenzae. These effects are potentially valuable in bacteriological investigation of suspected cases of pertussis, since the swabs received are often heavily infected with $H$. influenzae.

In some instances the incorporation of Difco proteose peptone $(1 \%)$ in meat extract horse blood medium resulted in complete inhibition of $\boldsymbol{H}$. pertussis while there was a particularly good growth of $H$. influenzae. The effect on the growth of $\boldsymbol{H}$. pertussis of heating this meat extract medium at $75^{\circ}$ was not marked; some slight deterioration was produced which disappeared when fresh serum was added. The growth of $\boldsymbol{H}$. influenzae was, however, much greater than in the unheated plates. Hence it is obvious that the failure of recently isolated strains of $\boldsymbol{H}$. pertussis to grow on heated blood agar is not due to a sensitivity to haematin compounds such as that shown by some strains of C. diphtheriae (Glass, $1939 a, b$ ).

We are indebted to the Medical Research Council for a contribution towards expenses and for a personal grant to one of us, and also to the students who cooperated in providing samples of blood. Dr D. E. Nicholson was working as an I.C.I. Research Fellow while engaged on this investigation.

\section{REFERENCES}

Barley, J. H. (1933). A medium for the isolation of Bacillus pertussis. J. inf. Dis. $52,94$.

Balch, H. H. (1942). Copper content of commercial peptones. J. Path. Bact. 54, 377.

Bordet, J. \& Gengou O. (1906). Le microbe de la coqueluche. Ann. Inst. Pasteur, 20,731 .

Chievitz, I. \& MeYer, A. (1918). Eine Methode zur Frühdiagnose des Keuchhustens. Münch med. Wschr. 2, 729.

Gardner, A. D. \& Leslie, P. H. (1932). Early diagnosis of whooping-cough by the cough-droplet method. Lancet, $\mathrm{i}, 9$.

Grass, V. (1939a). The effect of blood digest and haem on the growth of C. diphtheriae. J. Path. Bact. 49, 549.

Grass, V. (1939b). The effect of blood treated by heat, acid or alkali on the growth of C. diphtheriae. J. Path. Bact. 48, 507. 
Groot, E. H. (1946). Investigation into the biologically important amino-acids in potato protein in connection with its nutritive value. Parts I, II, III, IV and V. Arch. néerl. Physiol. 28, 277, 323, 340, 349, 357.

Hornibrook, J. W. (1939). Cultivation of Phase I $\boldsymbol{H}$. pertussis in a semi-synthetic medium. Pub. Hlth Rep., Wash., 54, 1847.

Hornibrook, J. W. (1940). Nicotinic acid as a growth factor for $H$. pertussis. Proc. Soc. exp. Biol., N.Y., 45, 598.

Kuernschmidt, H. (1931). Der Bordet-Gengousche Keuchhustenbacillus und der Pfeiffersche Influenzabacillus. Klin. Wschr. 2, 1847.

Kristensen, M. (1922). Investigations into the Occurrence and Classification of the Haemoglobinophilic Bacteria, p. 211. Copenhagen: Levin and Munksgaard.

Lawson, G. McL. (1939). Immunity Studies in pertussis. Amer. J. Hyg. 29, 119, section $B$.

Leslie, P. H. \& Gardner, A. D. (1931). The phases of Haemophilus pertussis. J. Hyg., Camb., 31, 423.

Mackie, T. J. \& McCartney, J. E. (1949). Handbook of Practical Bacteriology, 8th ed. p. 183. Edinburgh: E. and S. Livingstone Ltd.

Madsen, Th. (1925). Whooping cough. Its bacteriology, diagnosis, prevention and treatment. Boston med. surg. J. 192, 50.

O'Meara, R. A. Q. \& Macsween, J. C. (1937). The influence of copper in peptones on the growth of certain pathogens in peptone broth. J. Path. Bact. 44, 225.

Pollock, M. R. (1947). The growth of H. pertussis on media without blood. Brit. J. exp. Path. 28, 295.

Sauer, L. W. \& Hambrecht, L. (1930). Whooping cough. Early diagnosis by the cough plate method. J. Amer. med. Ass. 95, 263.

Silverthorne, N. \& Cameron, C. (1942). Whooping cough. IV. Beef liver extract agar media for growth of $\boldsymbol{H}$. pertussis. J. Pediat. 20, 16.

Straker, E. A. \& Westwater, J. S. (1937). The bacteriological diagnosis of whooping-cough. Lancet, ii, 565.

Sugare, H. \& McLeod, J. W. (1929). 'The bacteriological diagnosis of whoopingcough. Lancet, ii, 165.

Verwey, W. F., Thiele, E. H., Sage, D. N. \& Schuchardt, L. F. (1949). A simplified liquid culture medium for the growth of $H$. pertussis. J. Bact. 58, 127.

Wollstern, M. (1909). The Bordet-Gengou bacillus of pertussis. J. exp. Med. 11, 41.

(Received 6 July 1950) 